\title{
Cytological, linkage and insecticide studies on a genetic sexing line in Anopheles stephensi Liston
}

\author{
A. S. Robinson and \\ Pham Van Lap*
}

Insect Genetics Unit, Research Institute ITAL, P.O. Box 48, 6700 AA Wageningen, The Netherlands.

T(Y - D1)35 is a genetic sexing line in Anopheles stephensi $(2 \mathrm{n}=6 ; X X+X Y \delta)$ based on the translocation of the dieldrin resistance gene to the male determining chromosome. A cytological analysis demonstrated the presence of a 3 chromosome multiple translocation in which part of the $\boldsymbol{Y}$ chromosome formed the differential segment. An analysis of egg karyotypes enabled the segregation behaviour of the complex to be determined and only alternate and adjacent I segregations could be demonstrated. The breakpoint of the translocation in chromosome 3 was located using polytene salivary gland preparations. Linkage studies using chromosome three markers revealed contrasting effects of the translocation on recombination in this chromosome. An insecticide treatment method, based on the exposure of newly emerged adults, was developed enabling large numbers of individuals to be treated for the production of only males.

\section{INTRODUCTION}

Anopheles stephensi Liston is an important malaria vector in the Indian sub-continent and it has been the subject of studies aimed at the development of genetic control strategies (Sakai et al., 1983; Sakai and Mahmood, 1985; van Heemert et al., 1983). Most of these strategies involve the release of mosquitoes into field populations with the aim of either population suppression or replacement. In the majority of cases there is a requirement for an all male release and there has been a considerable effort put into the construction of genetic sexing systems (Lines and Curtis, 1985). In other mosquitoes, genetic sexing systems have been readily produced (Curtis et al., 1976; Curtis, 1978; Seawright et al., 1978; Baker et al., 1981; McDonald and Asman, 1982). In all these cases the translocation of an insecticide resistance gene to the male determining chromosome was the basis of the genetic sexing (Robinson, 1983).

In An. stephensi a genetic sexing system has recently been developed based on translocation of dieldrin resistance to the male-determining chromosome (Robinson, 1986). The present paper provides cytological confirmation of the transloca-

* Present address: Department of Genetics, Univ. of Hanoi, Vietnam. tion with cytological analysis of eggs enabling the segregation behaviour of the translocation complex to be elucidated. Analysis of the polytene chromosomes enabled the position of the dieldrin resistance locus to be determined and 3 point crosses allowed the effect of the translocation on crossing-over to be observed.

The insecticide treatment used to isolate the genetic sexing line initially resulted in significant mortality during adult emergence (Robinson, 1986). It was therefore necessary to develop a more efficient, practical and simple technique which could be used as a sexing method for large populations.

\section{MATERIALS AND METHODS}

\section{Strains}

-LSS-"London susceptible" homozygous susceptible for dieldrin and DDT (see Malcolm, 1986).

-DLDDT-homozygous resistant for dieldrin and DDT. Selected from matings between Lahore (Robinson et al., 1986) and Bar (obtained from London School of Hygiene and Tropical Medicine).

- T(Y-DL)35-genetic sexing line (Robinson, 1986). 
- M2-homozygous for chromosome 3 markers black larvae (B1), short palpi (sp) and dieldrin susceptible.

- $d p$-homozygous for chromosome 3 marker diamond palpus.

\section{Rearing}

All mosquitoes were held at $28^{\circ} \mathrm{C}$ and 80 per cent R.H. Larvae were fed on fish food (Tetramicromin) and adult blood meals were provided by anaesthetised mice.

\section{Cytology}

Mitotic and meiotic chromosomes were prepared from testes of 4th instar larvae as described by French et al. (1962). Salivary glands of fourth instar larvae were dissected in a droplet of 5 per cent diluted Carnoy's fixative and then transferred into a small drop of concentrated Carnoy's [3 ethyl alcohol: 1 acetic acid] on a siliconised coverslip. This tissue was stained with 2 per cent lacto-acetoorcein. The squashed preparations were sealed using photo glue and stored at $2^{\circ} \mathrm{C}$. Chromosomes were investigated under Carl Zeiss microscope. The salivary map of Sharma et al. (1969) was used for identification of the breakpoint. Eggs 6-8 hours were used for the preparation of chromosomes. The chorion was removed and the embryo stained in 2 per cent lacto-aceto-orcein for $1 \mathrm{~h}$. The tissue was then squashed in 45 per cent acetic acid.

\section{Insecticide treatment}

WHO test kits with 0.4 per cent dieldrin papers were used. The adult mosquitoes ( $\pm 24 \mathrm{~h}$ old $)$ were exposed for $2 \mathrm{~h}$ and mortality subsequently scored. Cone method 0.4 per cent dieldrin papers were shaped into the form of a cone with a hole in the top. The cone was placed above a circular dish containing pupae and the unit was placed in a cage. Two days after emergence the number and sex of live and dead mosquitoes in the cage, was noted.

\section{RESULTS}

\section{Cytological studies on $T(Y-D 1) 35$}

Metaphase preparation of testes from males in the genetic sexing line revealed in all cases an unusual karyotype in which all three chromosomes were involved in a translocation complex (fig. 1). Squashing too forcefully resulted in the dislocation of the complex into rings and chains. Analysis of
Figure 1 Metaphase I preparation from testes of line $T(Y$ DL) 35 in An. stephensi.

mitotic chromosomes (fig. 2) enabled the complex to be described in terms of chromosome breaks and exchanged segments. Irradiation had produced four chromosomal breaks, two in the $Y$ chromosome with one in each arm, one in $3 L$ and one in chromosome 2 . These are shown schematically in fig. 3. There was an unequal exchange between 3 and $Y$ with 3 losing a long segment and receiving a small segment from the long arm of $Y$. The translocated arm of 3 was therefore about one-third shorter than its normal homologue and the long arm of $Y$ because longer than normal; it even exceeded the length of the long arm of the $X$ chromosome. The second unequal exchange involved chromosome 2 and the short arm of $Y$. As a result chromosome 2 became shorter and the short arm of $Y$ became longer. This type of translocation therefore represents a 3 chromosome double translocation with the differential segment consisting of the centromeric portion of the $Y$ chromosome (see fig. 3).

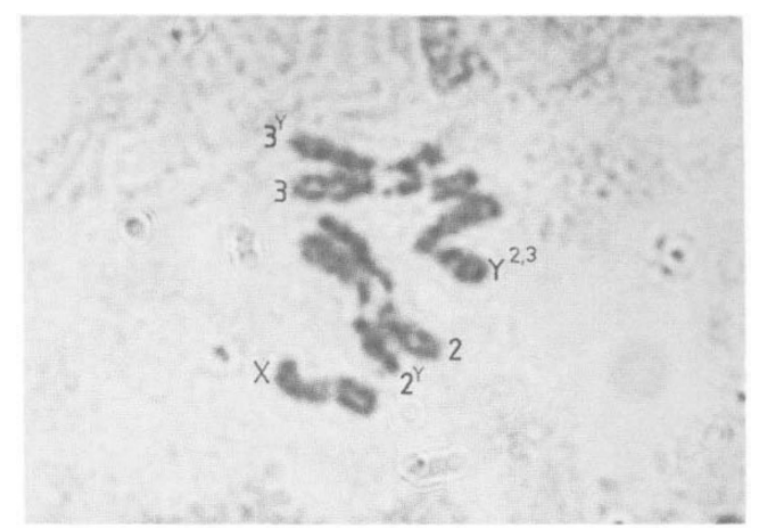

Figure 2 Mitotic chromosomes from testes in males of line $T(Y-D I) 35$ in An. stephensi. 


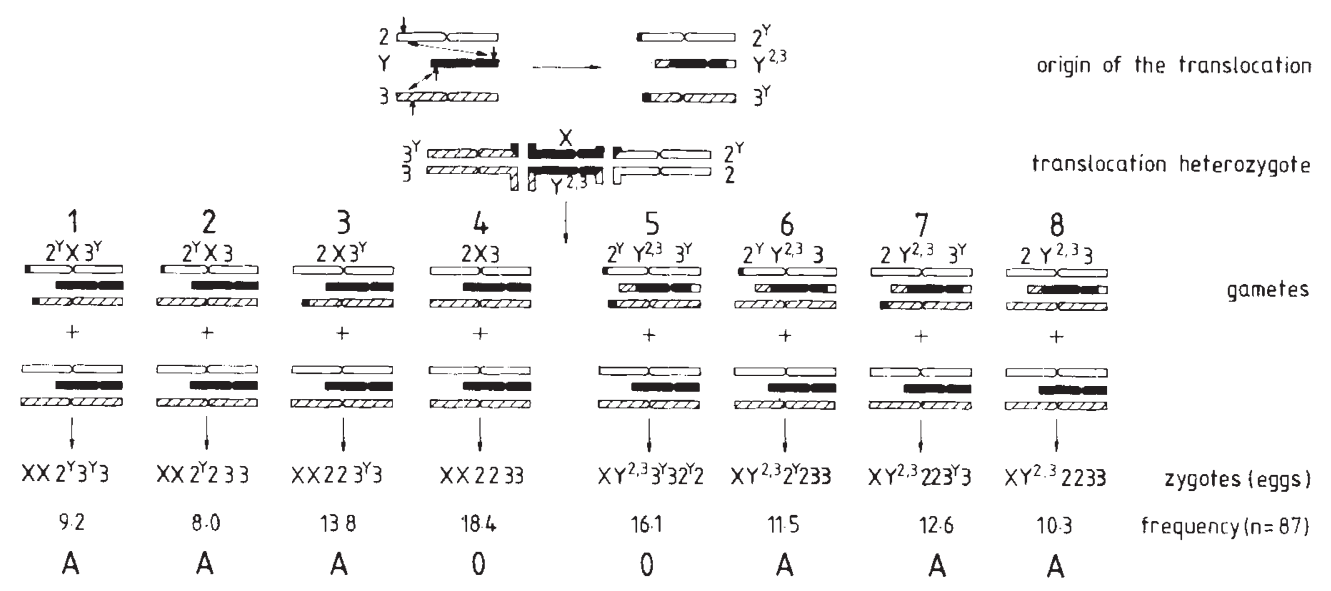

Figure 3 Schematic diagram showing the origin of the translocation complex and the observed segregants from the translocation complex ( $A$ represent aneuploid gametes and zygotes and $O$ represent orthoploid gametes and zygotes).

As the $Y$ chromosome received a long segment from chromosome 3 , synapsis was almost always observed between the long arm of $Y$ and the normal 3 (see fig. 2). The small exchanged segments between 2 and the short arm of $Y$ were unable to support homologous pairing in mitotic metaphase. However in prophase in eggs, pairing between 2 and the short arm of $Y$ could be seen. Due to the fact that both arms of the $Y$ chromosome were involved in the translocation there was very little somatic pairing between the sex chromosomes.

Analysis of polytene salivary gland chromosomes showed a complement composed of $5 \mathrm{arms}$, the shortest being the $X$ which was often separated from the chromocentre. The $Y$ chromosome is heterochromatic and does not polytenize. However by searching for discontinuities in the autosomes the position of the $Y$ /autosome breaks could be established. The polytene map of Sharma et al. (1969) was used to designate the chromosome break. In fig. 4 can be seen arms $2 L$ and $3 L$ and at position $43 / 44$ discontinuity can be seen indicating that the break in $3 L$ is at this position. As the dieldrin locus must be tightly linked to the translocation breakpoint it is concluded the gene coding for dieldrin resistance is located in $3 L$ very close to map reference 43/44. Sakai et al. (1983) using autosomal translocations suggested that the dieldrin locus was indeed in $3 L$. This has now been confirmed.

No discontinuity could be seen in either arm of chromosome 2 in the polytene preparation and it is not therefore clear where the break is. This suggests that there is a heterochromatic segment in the telomere region of this chromosome and that the break is in this region. Sakai et al. (1983) had some evidence for heterochromatin in $2 R$.

Using cytological analysis of mitotic chromosomes in young embryos (Vosselman and Van Heemert, 1980) an investigation was made of the segregation behaviour of the translocation complex. Fig. 3 shows the mitotic configurations observed in the eggs. Only alternate and adjacent I segregation were found i.e., homologous centromers always co-orient. Therefore the observed lack of pairing in mitotic divisions between the $X$ and $Y$ is not repeated under meitotic conditions.

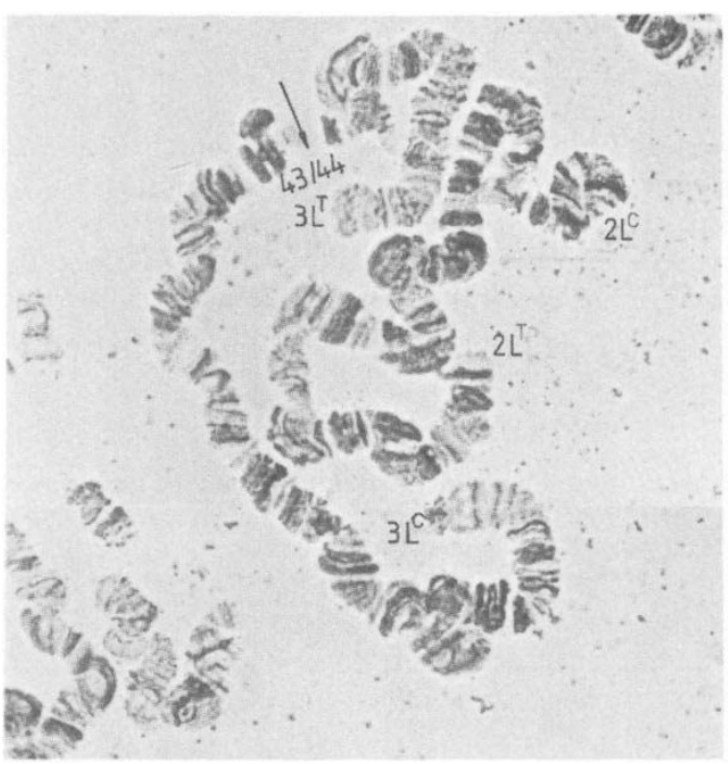

Figure 4 Salivary gland preparation in line $T(Y-D L) 35$ showing position of a single breakpoint in $3 L$ at $43 A$. 
The observed frequencies of the eight types are also given in fig. 3 . The orthoploid types ( 4 and 5) constitute 32 per cent of the embryos scored therefore the expected fertility is higher than that observed ( 23 per cent). This discrepancy can perhaps be explained by the fact that types 2 and 4 and 5 and 7 are very difficult to distinguish from each other. The only difference being that chromosome 2 is slightly shorter than normal.

\section{Linkage data}

Linkage was measured between the breakpoint in $3 L$ and three chromosome 3 markers, black larvae (B1), short palpi ( $s p)$ and diamond palpi $(d p)$, to answer the following questions. Does this rather complex rearrangement affect the recombination in general and in particular, does the presence of a translocation breakpoint in chromosome 3 , very close to the dieldrin locus, affect recombination between the dieldrin locus and its closest known marker, B1? The gene order was reported by Parvez et al. (1985) to be $s p-d p-B 1-D L$ with free recombination between $s p$ and $B 1$ and 22 per cent recombination between $B 1$ and $D 1$. The results are shown in table 1 . The average recombination rate between $s p$ and $B 1$ was 38 per cent significantly lower $\left(\chi_{1}^{2}=25 \cdot 6^{* * *}\right)$ than that recorded by Akhtar et al. (1982) while that between $D 1$ (the breakpoint $)$ and $B 1$ was significantly higher $\left(\chi_{1}^{2}=\right.$ $\left.6.45^{*}\right)$ than has been found Akhtar et al. (1982). This translocation ther would appear to be increasing recombination in the region $D 1$ and $B 1$ and suppressing recombination between $s p$ and $B 1$ a rather paradoxical situation. In a separate experiment, recombination between the breakpoint and $d p$ was found to be 38 per cent, which is almost identical to that found for the $d p-D 1$ interval by Akhtar et al. (1982). The crossover values have been interpreted on the assumption that the translocation breakpoint is genetically inseparable from the D1 locus based on the stability of the linkage of sex with dieldrin for 25 generations of mass rearing.

\section{Insecticide Studies on $T(Y-D L) 35$ adults:}

The method to select $T(Y-D L) 35$ as a genetic sexing line was a fourth instar larval treatment but this led to unacceptable levels of mortality during adult emergence which would be unacceptable during the planned large scale release of males. Treatment of first instar larvae was attempted but discrimination was inadequate and the test was even more difficult to carry out than fourth instar treatment. Adult treatments were next considered. Standard WHO test kits were used with 0.4 per cent dieldrin papers. Adults from the $T(Y-D L) 35$ line were exposed to the insecticide for 2 hours, transferred from the exposure tube to the recovery tube and mortality was checked regularly. Using adults which were 1 or 2 days old excellent discrimination between the sexes was found with 100 per cent female mortality after about 3 hours (table 2 ). When the experiments were repeated with 7 day old adults almost all the females survived (table 2). It appears that adult age is a critical factor and it has very clear effect on the susceptibility of females to dieldrin.

The observation that following emergence from the pupae mosquitoes need a period of rest stimulated the development of the "cone" technique (see Materials and Methods). The results of the test of this technique can be seen in table 3 . Initially the homozygous susceptible and resistant strains were tested. The susceptible strain (LSS) showed almost complete mortality, only 8 females were found alive from a total of 2510 mosquitoes tested. With DLDDT the opposite picture was produced with 97 per cent of individuals alive 2 days after emergence from the cone. With $T(Y-D 1) 35$ excellent discrimination was achieved with only 4 females out of 643 surviving the treatment. It appears that during the resting phase, (the duration

Table 1. Recombination between $B l, s p$ and the translocation breakpoint in strain $T(Y-D L) 35$ in An. stephensi. T( Y-Dl)35 double heterozygotes for $B l$ and $s p$ (with the mutants in coupling but in repulsion to the translocation) were backcrossed to females homozygous for $B l$ and $s p$

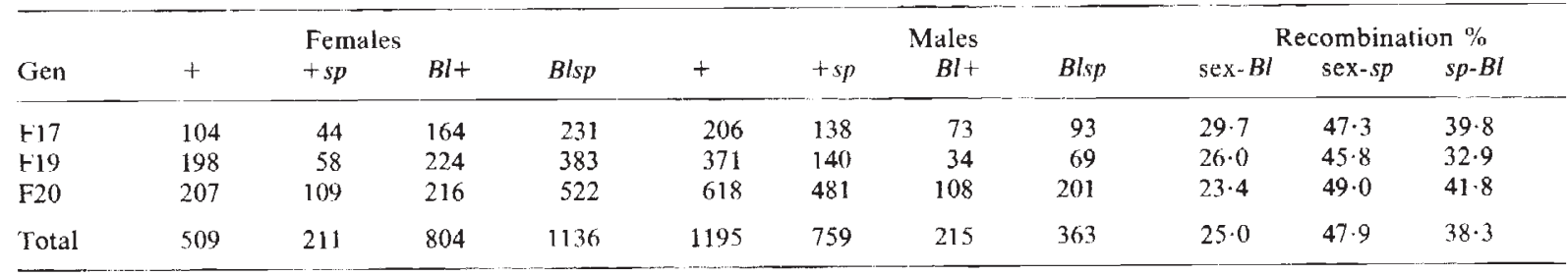


Table 2 Effect of dieldrin on adult An. stephensi survival after 2 hours exposure to 0.4 per cent papers in W.H.O. test kits. No. of hours refers to time after completion of the insecticide treatment

\begin{tabular}{|c|c|c|c|c|c|c|c|c|c|c|c|c|}
\hline \multirow{2}{*}{\multicolumn{2}{|c|}{$\begin{array}{l}\text { No. and } \\
\text { sex of } \\
\text { Mosquitos }\end{array}$}} & \multirow{3}{*}{$\begin{array}{l}\text { Age } \\
\text { (Days) }\end{array}$} & \multicolumn{10}{|c|}{$\begin{array}{l}\text { No. mosquitoes alive at indicated no. of hours } \\
\text { after insecticide treatment }\end{array}$} \\
\hline & & & \multicolumn{2}{|c|}{$1 \mathrm{~h}$} & \multicolumn{2}{|c|}{$2 \mathrm{~h}$} & \multicolumn{2}{|c|}{$2 \cdot 5 \mathrm{~h}$} & \multicolumn{2}{|c|}{$3 \cdot 5 \mathrm{~h}$} & \multicolumn{2}{|c|}{$4 \cdot 0 \mathrm{~h}$} \\
\hline$\delta$ & $q$ & & $\delta$ & q & $\delta$ & $q$ & 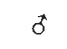 & q & $\sigma$ & $q$ & $\delta$ & q \\
\hline 87 & 57 & 1 & 87 & 57 & 86 & 21 & 85 & 4 & 85 & 0 & 85 & 0 \\
\hline 53 & 84 & 1 & 53 & 83 & 53 & 63 & 53 & 14 & 51 & 6 & 51 & 0 \\
\hline 60 & 65 & 2 & 60 & 65 & 59 & 34 & 54 & 2 & 59 & 0 & 54 & 0 \\
\hline 85 & 87 & 2 & 83 & 86 & 82 & 67 & 82 & 11 & 82 & 1 & 82 & 0 \\
\hline$\overline{284}$ & $\overline{293}$ & - & $\overline{283}$ & $\overline{291}$ & $\overline{280}$ & $\overline{140}$ & $\overline{279}$ & $\overline{31}$ & $\overline{277}$ & $\sqrt{7}$ & $\overline{277}$ & $\overline{0}$ \\
\hline 35 & 19 & 7 & 35 & 17 & 35 & 17 & 35 & 16 & 34 & 16 & & \\
\hline
\end{tabular}

Table 3 Effect of dieldrin on newly-emerged An. stephensi mosquitoes using the "cone" method. Mosquitoes were counted 2 days after emergence

\begin{tabular}{|c|c|c|c|c|c|c|}
\hline \multirow{3}{*}{ Strain } & \multicolumn{2}{|c|}{ No. pupae } & \multicolumn{4}{|c|}{ No. mosquitoes } \\
\hline & \multirow{2}{*}{ tested } & \multirow[t]{2}{*}{ dead } & \multicolumn{2}{|c|}{ alive } & \multicolumn{2}{|c|}{ dead } \\
\hline & & & o & $q$ & o & $q$ \\
\hline LSS & 2510 & 0 & 0 & 8 & 1234 & 1268 \\
\hline DLDDT & 1060 & 93 & 450 & 487 & 11 & 19 \\
\hline$T(Y-D L) 35$ & 1324 & 35 & 639 & 4 & 6 & 640 \\
\hline
\end{tabular}

of which has not been determined), sufficient insecticide is picked up in order to discriminate between homozygous susceptible individuals and heterozygotes. The "cone" method is at present being used in large population cage experiments in which large numbers of males of this line are required.

\section{DISCUSSION}

$T(Y-D L) 35$ was found to carry a three chromosome double translocation of the type $3 a$ described by Curtis and Robinson (1971) in which the centromere of the $Y$ chromosome is located in the differential segment. The break in chromosome 3 is located at or very near the dieldrin locus which is at position $43 / 44$ as determined by polytene analysis. Using other male-linked translocations the loci $B 1$ and $s p$ have now been assigned to their position on the polytene chromosome (Lap and Robinson unpublished results).

A three chromosome double translocation with a differential segment is in general vulnerable to breakdown due to recombination occurring in the differential segment thus generating novel chromosomes. This was demonstrated in a double translocation in Aedes aegypti (McDonald and Rai, 1970).
In the present case where the $Y$ chromosome is involved in the differential segment the situation is somewhat different as crossing-over probably does not occur between the sex chromosomes in this species as male linked translocations remain so linked through many generations of rearing. This indicates why this translocation shows such impressive stability. In a closely related species, An. culicifacies, crossing-over does occur between the sex chromosomes (Sakai et al., 1979) and therefore such a translocation as this would be subject to breakdown.

As well as the translocation showing stability, the linkage of the dieldrin resistance gene to the male sex appears also to be stable as the line has been reared for about 25 generations in the laboratory without selection. The only breakdown in the strain was traced to contamination resulting from a technical error. This indicates that the translocation breakpoint and the dieldrin locus are extremely closely linked. However, it proved impossible to sex this line using WHO test kits when adults were 7 days old as the females showed limited mortality. This age effect of dieldrin has not been demonstrated previously, however, with malathion an age effect has been demonstrated (Rowland, 1985). 
Analysis of young embryos enables translocation segregation to be assessed in the absence of adequate meiotic configurations (Vosselman and van Heemert, 1980). Adjacent II segregation (i.e., co-operation of non-homologous centromers) was not observed and the frequency of alternate segregants was 0.32 which should have led to an equivalent egg hatch frequency. The observed egg hatch was 23 per cent (Robinson, 1986), significantly lower, $\left(\chi_{1}^{2}=6 \cdot 25^{*}\right)$ the reason for this discrepancy can probably be found in the misclassification of certain of the segregation types.

This multiple translocation had contrasting effects on linkage values in chromosome 3 . It appeared that the translocation was reducing recombination in chromosome regions distant from the breakpoint i.e., between $s p$ and $B 1$ while increasing recombination in regions close to the breakpoint i.e., between the breakpoint and $B 1$. In many studies in Drosophila, translocations have been shown to be very effective cross-over suppressors although these effects on crossing-over do depend on the position of the chromosome break. In general breaks in the middle of chromosome arms tend to reduce crossing-over the most (Roberts 1976) and breaks at the tip or base of the chromosome have very little effect on recombination. In the present case there appears to be a compensating effect between the two chromosome regions with the overall effect of the translocation being negligible. However, recombinations rates in general do vary between strains, it is therefore possible that the significant differences reported here could be due to such strain differences. This has been demonstrated in An. stephensi (Robinson et al., 1986).

The development of the cone method for the treatment of newly emerged adults of this strain enables large numbers of individuals to be treated and it should present no problems to integrate this method into a mass rearing system producing males for field release. The incorporation of genetic sexing systems into mass rearing facilities has already been demonstrated to be successful in $A n$. albimanus (Bailey et al., 1980) and in Lucilia cuprina (Foster et al., 1978), however, in the latter case the males showed reduced fitness in the field. The present strain is homozygous susceptible for malathion and it has been selected for DDT susceptibility (Mali, unpublished results). It will be used to try to introduce susceptibility for these two insecticides into a resistant population though it is recognised that this strain has a disadvantage due to its low fertility. The $Y$ translocation will not "drive" the replacement of the population but release of only males is imperative when field releases are being considered. Large insecticide resistant laboratory populations have now been established and releases of males only using the cone method will be used to try to introduce insecticide susceptibility.

Acknowledgements This research is financially supported by the Dutch Ministries of Agriculture and Fisheries, Developmental Aid and Science Policy and forms part of a collaborative programme between the Research Institute ITAL and Departments of Parasitology in Amsterdam, Nijmegen and Leiden. Wiun van den Brink, Angela Heemskerk, Pieternel Mali and Gijs Schelling are thanked for the team effort. Pham Van Lap was in receipt of a NUFFIC fellowship.

\section{REFERENCES}

AHKTAR, K, SAKAI, R. K. AND BAKER, R. H. 1982. Linkage group III in the malaria vector, Anopheles stephensi. $J$ Hered., 73, 473-475.

BAILEY, 1). L., LOWE, R. E., DAML, D. A. AND SEAWRIGHI, I. A. I980. Mass rearing the genetically altered MAC $\mathrm{HO}$ strain of Anopheles albaminus Wiedemann. Amer. J. Trop. Med. Hyg., 29, 14I-149.

BAKER, R. H., SAKAI, R. K. ANI) RAANA, K. 1981. Genetic sexing for a mosquito sterile-male release. $J$. Hered., 12, 216-218.

CuRtis, C. F. 1978. Genetic sex separation in Anopheles arabiensis and the production of sterile hybrids. Bull W.H.O., 56, 453-454.

CURTIS, C F. AND ROBINSON, A. S I97I. Computer simulation of the use of double translocations for pest control. Genetics, 69, 97-113.

CURTIS, C. F, AKIYAMA, J. AND DAVIDSON, G. 1976. A genetic sexing system in Anopheles gambiae species A. Mosq. News, $36,492-498$

FOSTER, (i. G., WHITTEN, M. S., VOGT, W. G., WOODBURN, T L. AND ARNOLD, S. T. 1978. Larval release method for genetic control of the Australian sheep blowfly, Lucilia cuprina (Wiedemann) (Diptera: Calliphoridae) Bull. ent Res., 68, 75-83.

FRINCH, W. L., BAKER, R. H. AND KITZMILIER, J. B. 1962 Preparation of mosquito chromosomes. Mosq. News, 22, 377-383.

HEEMERT, C. VAN, TOAN, T. A., ROBINSON, A. S. AND FFLD MANN, A. 1983. Induction and isolation of translocations in Anopheles stephensi. Mosq. News., 43, 480-484.

IINES, J. AND CURTIS, C. F. 1985. Genetic sexing systems in Anopheles arabiensis Patton. J. econ. Entomol., 78, 848-851.

MALCOLM, C. Genetic analysis of reduced susceptibility to knockdown by permethrin and its relationship to DDT resistance in larvae of Anopheles stephensi. Bull. Ent. Res. (submitted).

MCDONALD, P. T. AND ASMAN, S. M. 1982. A genetic sexing strain based on malathion resistance for Culex tarsalis. Mosq. News, 42, 531-536.

MCDONALD, P. T. AND RAi, K. S. 1970. Aedes aegypti: Origin of a "new" chromosome from a double translocation heterozygote. Science, 168, 1229-1230.

PARVEZ, S. D., AKHTAR, K. ANI SAKAI, R, K. 1985. Two new mutations and a linkage map of Anopheles stephensi. $J$. Hered., 76, 205-207. 
ROBERTS, P. A. 1976. The genetics of chromosome aberration. Ashburner, M. and Novitski, E. (eds.). In The Genetics and Biology of Drosophila. Vol. la, Academic Press Inc. (London) Ltd, p. 486.

ROBINSON, A. S. 1983. Sex-ratio manipulation in relation to insect pest control. Annu. Rev. Genetics, 17, 191-214.

Robinson, A. S. 1986. Genetic sexing in Anopheles stephensi using dieldrin resistance. J. Amer. Mosq. Cont. Assoc., 2, 93-95.

ROBINSON, A. S., MALCOLM, C., MALI, P. AND SCHELLING, G. 1986. Breakpoint distribution in male-linked translocations in Anopheles stephensi Liston. J. Hered. In press.

ROWLAND, M. 1985. Location of the gene for malathion resistance in Anopheles stephensi (Diptera: Culicidae) for Pakistan. J. Med. Entomol., 22, 373-380.

SAKAI, R. K. AND MAHMOOD, F. 1985. Homozygous chromosomal aberrations in Anopheles stephensi, J. Hered., 76, $230-236$.
SAKAI, R. K., BAKER, R. H., RAANA, K. AND HASSAN, M. 1979 Crossing-over in the long arm of the $X$ and $Y$ chromosomes in Anopheles culicifacies. Chromosoma, 14, 204-218.

SAKAI, R. K., MAHMOOD F., AKHTAR, K., DUBASH, C. T. AND BAKER, R. H. 1983. Induced chromosomal aberrations and linkage group-chromosome correlation in Anopheles stephensi. J. Her.ed, 14, 232-238.

SEAWRIGHT, J. A., KAISER, P. E., DAME, D. A. AND LOFGREN, C. S. 1978. Genetic method for the preferential elimination of females of Anopheles albimanus. Science, 220, 13031304.

SHARMA, G. P., PARSHAD, R., NARANG, S. L. AND KITZMILLER, J. B. 1969. The salvary chromosomes of Anopheles stephensi. J. Med. Entomol, 6, 68-71.

vosSELMAN, L. AND VAN HEEMERT, C. 1980. Meiotic disjunction and embryonic lethality in sex-linked double-translocation heterozygous males of the onion fly, Hylemya antiqua. (Meigen). Theoret. appl. Genet., 58, 161-167. 